\title{
Engineering CRISPR interference system in Klebsiella pneumoniae for attenuating lactic acid synthesis
}

Jingxuan Wang ${ }^{1}$, Peng Zhao ${ }^{1}$, Ying $\mathrm{Li}^{2}$, Lida Xu ${ }^{1}$ and Pingfang Tian ${ }^{1 *}$

\begin{abstract}
Background: Klebsiella pneumoniae is a promising industrial species for bioproduction of bulk chemicals such as 1,3-propanediol, 2,3-butanediol and 3-hydroxypropionic acid (3-HP). However, lactic acid is a troublesome by-product when optimizing for 3-HP production. Therefore, it is highly desirable to minimize lactic acid.

Results: Here, we show that lactic acid synthesis can be largely blocked by an engineered CRISPR interference (CRISPRi) system in K. pneumoniae. EGFP was recruited as a reporter of this CRISPRi system. Fluorescence assay of this CRISPRi system showed that enhanced green fluorescent protein (EGFP) expression level was repressed by $85-90 \%$. To further test this CRISPRi system, guide RNAs were designed to individually or simultaneously target four lactate-producing enzyme genes. Results showed that all lactate-producing enzyme genes were significantly repressed. Notably, D-lactate dehydrogenase (IdhA) was shown to be the most influential enzyme for lactic acid formation in micro-aerobic conditions, as inhibiting IdhA alone led to lactic acid level similar to simultaneously repressing four genes. In shake flask cultivation, the strain coexpressing puuC (an aldehyde dehydrogenase catalyzing 3-hydroxypropionaldehyde to 3-HP) and dCas9-sgRNA inhibiting IdhA produced 1.37-fold 3-HP relative to the reference strain. Furthermore, in bioreactor cultivation, this CRISPRi strain inhibiting $/ \mathrm{dhA}$ produced $36.7 \mathrm{~g} / \mathrm{L} 3-\mathrm{HP}$, but only generated $1 \mathrm{~g} / \mathrm{L}$ lactic acid. Clearly, this engineered CRISPRi system largely simplified downstream separation of 3-HP from its isomer lactic acid, an extreme challenge for 3-HP bioprocess.
\end{abstract}

Conclusions: This study offers a deep understanding of lactic acid metabolism in diverse species, and we believe that this CRISPRi system will facilitate biomanufacturing and functional genome studies of K. pneumoniae or beyond.

Keywords: CRISPR interference, Klebsiella pneumoniae, 3-Hydroxypropionic acid, Lactic acid, Lactate dehydrogenase

\section{Background}

For bioproduction of chemicals, byproducts are problematic because they not only consume cellular resources but also entangle downstream separation. Conventional genetic engineering strategies to attenuate byproducts formation mainly rely on deletion or repression of their biosynthesis genes [1-4]. However, these approaches in most cases compromise cell growth which in turn hampers the production of desired metabolites [5]. In recent

\footnotetext{
${ }^{*}$ Correspondence: tianpf@mail.buct.edu.cn

${ }^{1}$ Beijing Key Laboratory of Bioprocess, College of Life Science and Technology, Beijing University of Chemical Technology, Beijing 100029, People's Republic of China

Full list of author information is available at the end of the article
}

years, Klebsiella pneumoniae has attracted much attention because it can naturally convert glycerol to a range of economically important bulk chemicals including 1,3-propanediol (1,3-PDO), 2,3-butanediol (2,3-BDO), 3-hydroxypropionic acid (3-HP) and D-lactic acid [1, 2, 6-11]. Glycerol metabolism in K. pneumoniae is mediated by the $d h a$ and $g l p$ regulons in anaerobic or microaerobic conditions $[6,9,12]$. The dha regulon involves glycerol reduction and oxidation pathways (Fig. 1). In the reduction pathways, glycerol is converted to 3-hydroxypropionaldehyde (3-HPA) by GDHt (encoded by dhaB cluster, GenBank No. U30903). Next, 3-HPA is converted to $1,3-\mathrm{PDO}$ by 1,3 -propanediol dehydrogenase (PDOR, encoded by dhaT) [13]. 3-HPA is also converted to 3-HP 


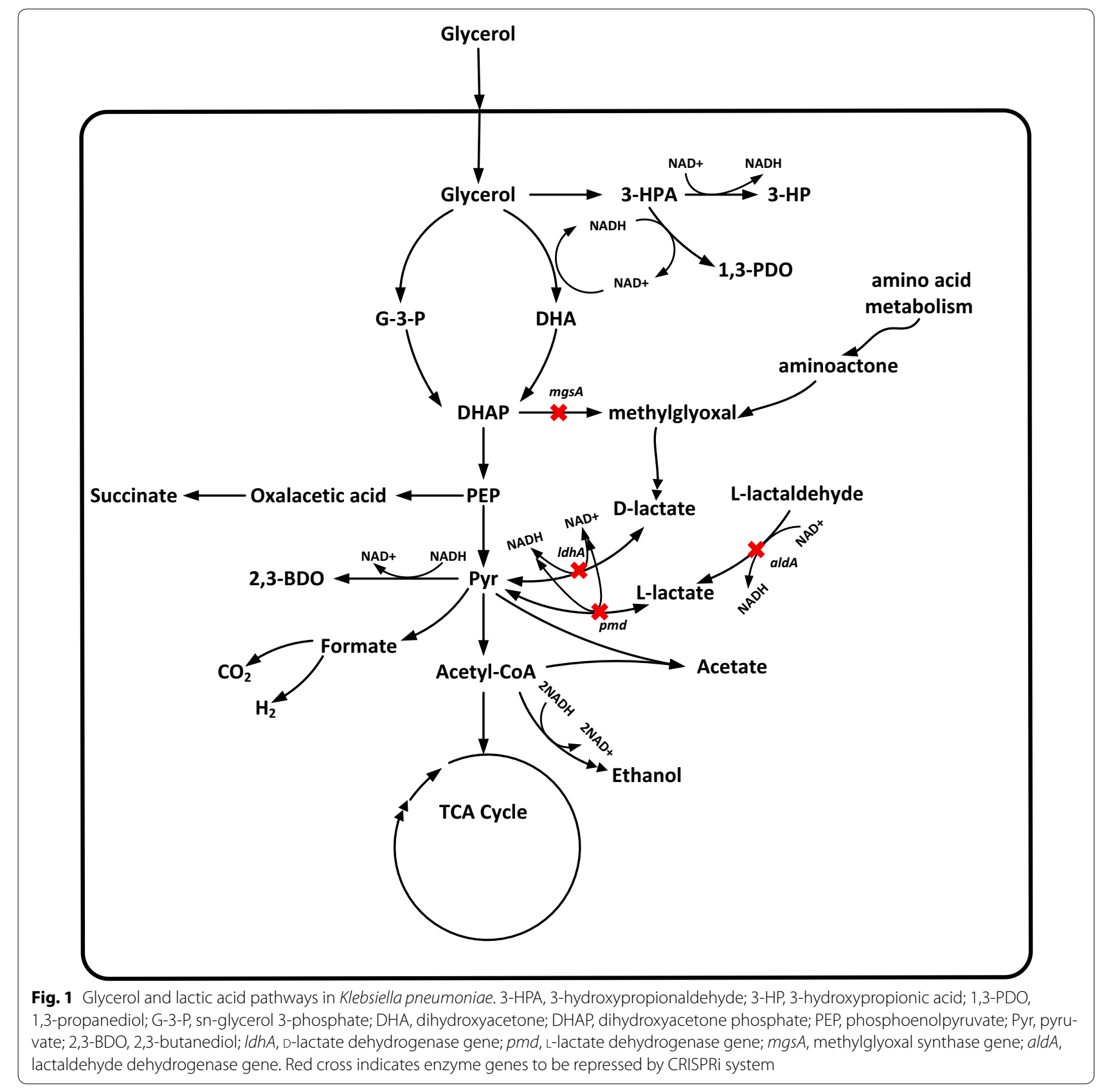

by aldehyde dehydrogenase (ALDH) with $\mathrm{NAD}^{+}$as a cofactor [9]. In the glycerol oxidation pathways, a series of metabolites such as ATP, $\mathrm{NAD}^{+}, 2,3-\mathrm{BDO}$ and lactic acid are generated to sustain cell growth and benefit 1,3PDO and 3-HP production [9]. Of various metabolites, lactic acid is the major byproduct in the production of 1,3-PDO, 2,3-BDO and 3-HP [14]. The formation of lactic acid not only consumes carbon source but also entangles downstream separation [2]. It is extremely challenging to separate lactic acid (2-hydroxy propionic acid) from 3-HP (3-hydroxy propionic acid) because they are isomers. On the other hand, although the formation of lactic acid consumes carbon flux, it also augments carbon flux toward glycerol reduction pathways. Especially, when dissolved oxygen is insufficient, lactic acid synthesis leads to $\mathrm{NAD}^{+}$ regeneration which in return drives $3-\mathrm{HP}$ production because $\mathrm{NAD}^{+}$is the cofactor of aldehyde dehydrogenase that catalyzes 3-HPA to 3-HP [7, 9]. In other words, 3-HP production consumes $\mathrm{NAD}^{+}$, and the lack of $\mathrm{NAD}^{+}$in turn necessitates lactic acid synthesis. Clearly, there is 
interdependence between lactic acid formation and 3-HP production. Hence, knockdown instead of deletion of lactic acid pathways is beneficial for 3-HP production.

CRISPR technology opens avenue for simultaneous knockdown or knockout of multiple genes due to an array of single guide RNAs (sgRNAs) that direct dCas9 or Cas9 to interference or edit target genes [15-19]. The dCas9sgRNA-based CRISPR interference (CRISPRi) tools mainly include CRISPR activation and CRISPR repression [20, 21]. The dCas9-sgRNA complex activates gene expression when dCas9 is fused with the omega subunit of RNA polymerase, while it represses gene expression when dCas9 binds a promoter or an open reading frame (ORF) [21]. The Cas9-based DNA cleavage largely relies on DNA repair mechanisms including homologous recombination in prokaryotes and non-homologous end joining (NHEJ) in eukaryotes [17]. Unlike Cas9-based DNA editing, CRISPRi is independent of DNA repair and thus can be easily applied in microbes lacking the NHEJ pathway or for which no efficient homologous recombination approach is available. Furthermore, compared with CRISPR editing that may lead to slowed cell growth or even cell death, CRISPRi could be more appropriate for modulating multiple genes.

In view of above information, we anticipated that CRISPRi may be ideal for modulating lactic acid metabolism which is subjected to multiple factors. To validate this prediction, in this study we developed CRISPRi system in K. pneumoniae. Detailed analysis of glycerol consumption, cell growth, metabolites formation and gene transcription was to systematically assess the performance of CRISPRi system in K. pneumoniae. Shake-flask and bioreactor cultivation of the recombinant $K$. pneumoniae strain harboring CRISPRi vectors (hereafter refers to as CRISPRi strain) were to determine the key enzymes affecting lactic acid synthesis. Overall, this study was to exploit CRISPRi system for basic research and metabolic engineering of K. pneumoniae.

\section{Results}

Fluorescence assay of dCas9-gRNA-mediated transcription repression in $K$. pneumoniae

The dCas9 was derived from Streptococcus pyogenes, and CRISPRi system was developed to dissect lactic acid metabolism. To determine whether it functioned in $K$. pneumoniae, EGFP was employed as a reporter under $t a c$ promoter. To ensure CRISPRi efficiency, two candidate guide RNAs $\mathrm{T}_{1}$ and $\mathrm{T}_{2}$ toward the different regions of tac promoter were designed and chemically synthesized. The CRISPRi vector with non-targeting sgRNA was used as a control. The fluorescence intensity of single strain was calculated as the total fluorescence intensity divided by $\mathrm{OD}_{600}$ value. Results showed that EGFP expression was significantly down-regulated by CRISPRi system (Fig. 2). Compared with the control strain NT with non-targeting guide RNA, strains T1 and T2 showed a remarkable decrease in the fluorescence intensity even if anhydrotetracycline (aTc) was absent. Strains $\mathrm{T}_{1}$ and $\mathrm{T}_{2}$ respectively exhibited 65 and 23\% inhibition on EGFP when aTc was absent. Notably, $\mathrm{T}_{1}$ and $\mathrm{T}_{2}$ respectively displayed 85 and 90\% inhibition on EGFP level when aTc was added into medium. Overall these results indicated that CRISPRi system significantly repressed EGFP expression, although tet promoter failed to tightly control dCas9 expression in K. pneumoniae. Namely, there existed leaky expression of dCas9.

\section{Repression of lactate-producing enzyme genes by CRISPRi system}

To further validate the CRISPRi system, it was harnessed to repress lactate-producing enzyme genes. In $K$. pneumoniae, many enzymes potentially contribute to lactic acid synthesis, including L-lactate dehydrogenase $(p m d)$, D-lactate dehydrogenase (ldhA), lactaldehyde dehydrogenase (aldA) and methylglyoxal (mgsA). To determine the key enzymes affecting lactic acid formation, an array of sgRNAs targeting enzyme genes were designed and chemically synthesized. For each lactate-producing enzyme gene, two or three candidate sgRNAs were subjected to screening. The sgRNAs were designed by using online software CRISPR direct (http://crispr.dbcls.jp/ doc/) [22]. In principle, sgRNA sequence should target transcription initiation site especially that in nontemplate strand [23]. In addition, sgRNA sequence was used as a query to search against $K$. pneumoniae

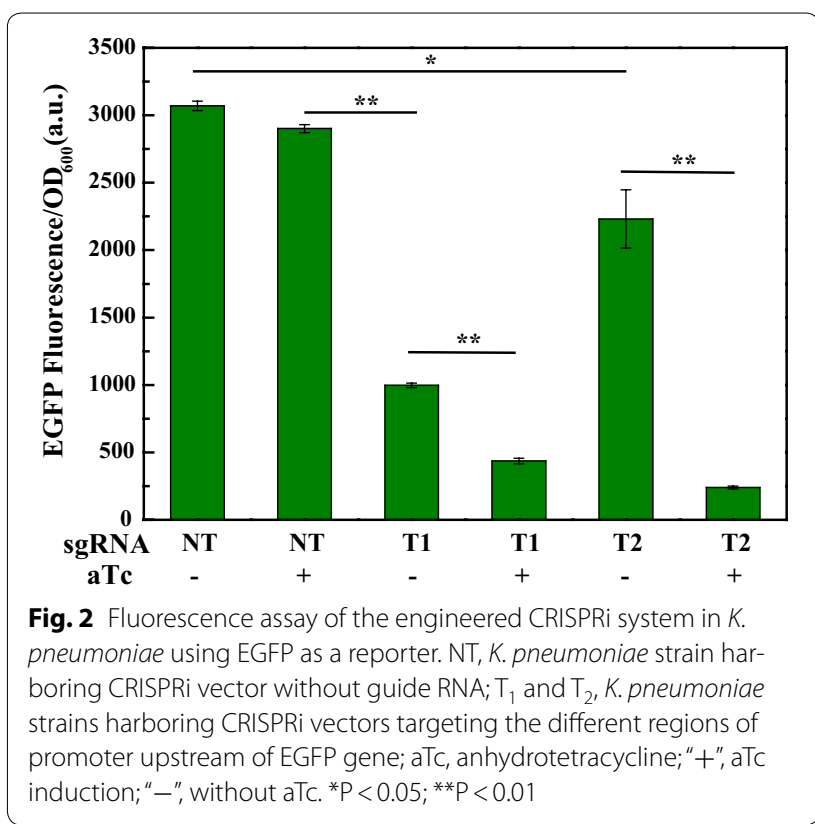


genome to avoid targeting homologous sequence and off-target. The secondary structure of sgRNAs were predicted by using online Quikfold algorithm from the UNAFold package [24]. All engineered vectors were confirmed by sequencing. The CRISPRi vectors targeting different lactate-producing enzyme genes were transformed into the previously engineered 3-HP-producing strain $\mathrm{Kp}($ ptac-puuC) [2], resulting in recombinant strain $\mathrm{Kp}($ ptac- $p u u C+$ placiL), Kp(ptac-puuC+ placiD), $\mathrm{Kp}($ ptac- $p u u C+$ placiA) and $\mathrm{Kp}($ ptac-puuC+placiM $)$. Next, quantitative real-time PCR (qRT-PCR) was performed to examine CRISPRi efficiency toward lactateproducing enzyme genes. Results showed that guide RNAs L1 and L2 failed to significantly inhibit pmd gene (Fig. 3a). However, for three CRISPRi strains targeting $l d h A$, ald $A$ and $m g s A$, at least one sgRNA showed inhibition activity (Fig. 3b-d). To simultaneously repress four lactate-producing enzyme genes, four well-functioned sgRNAs (L3 for $p m d$, D1 for ldhA, A2 for aldA, M2 for $m g s A$ ) were joined together to form a new vector named placiMALD. To investigate the performance of CRISPRi system, we engineered a vector named Kp(ptacpuuC+ placiMALD), where vectors dCas9-sgRNA and puuC were coexpressed. The qRT-PCR assay showed that CRISPRi vector placiMALD significantly repressed all lactate-producing enzyme genes (Fig. 3e).

\section{Shake flask cultivation of the CRISPRi strains}

To overproduce 3-HP and attenuate lactic acid formation, PuuC was coexpressed with dCas9. To do so, we engineered five recombinant strains designated $\mathrm{Kp}(\mathrm{ptac}-$ puuC + placiL $), \quad \mathrm{Kp}($ ptac-puuC + placiD $)$, $\mathrm{Kp}(\mathrm{ptac}-p u u C+$ placiA $), \quad \mathrm{Kp}($ ptac-puuC + placiM $)$ and $\mathrm{Kp}(\mathrm{ptac}-\mathrm{puuC}+$ placiMALD). These strains were fermented in micro-aerobic conditions with $\mathrm{Kp}(\mathrm{ptac}-$ puu $C+$ pdCas9) as the control. In $15 \mathrm{~h}$ cultivation, these strains showed retarded growth and postponed log phase with exception of the strain $\mathrm{Kp}($ ptac-puuC+placiM), where "placiM" indicates CRISPRi vector targeting $m g s A$ gene (Fig. 4a). Interestingly, in stationary phase, all these strains demonstrated similar $\mathrm{OD}_{600}$ value with the control $\mathrm{Kp}($ ptac-puuC+pdCas9). However, due to harboring of two vectors $\mathrm{pSg}$ and ptac-puuC+Cas9, glycerol consumption was largely retarded (Fig. 4b). For lactic acid formation, the strain harboring CRISPRi vector targeting $m g s A$ had highest lactic acid peak (Fig. 4c). In contrast, the strains $\mathrm{Kp}($ ptac-puuC + placiD) and $\mathrm{Kp}(\mathrm{ptac}-\mathrm{puuC}+$ placiMALD) produced less lactic acid (Fig. 4c). For other strains, lactic acid accumulated in $\log$ phase but declined in stationary phase. In addition, strains $\mathrm{Kp}(\mathrm{ptac}-$ puuC+placiD) and $\mathrm{Kp}$ (ptacpuuC+ placiMALD) produced more $3-\mathrm{HP}$ and 1,3$\mathrm{PDO}$ at $30 \mathrm{~h}$ than other strains did. Only the strain
$\mathrm{Kp}($ ptac-puuC + placiM) produced less $3-\mathrm{HP}$ than the control. For acetic acid and 2,3-BDO production, no significant difference was observed in all strains (Fig. 4d).

\section{Fed-batch cultivation of CRISPRi strains}

As mentioned, the strains $\mathrm{Kp}($ ptac-puuC + placiD) and $\mathrm{Kp}(\mathrm{ptac}-$ puuC + placiMALD) produced less lactic acid in shake flasks relative to other strains (Fig. 4c). To further investigate the performance of CRISPRi system, the above two strains were independently cultivated in $5 \mathrm{~L}$ bioreactor. Results showed that only trace amount of lactic acid was generated during entire fermentation process (Fig. 5b, c). In addition, due to PuuC overexpression, the strain $\mathrm{Kp}($ ptac-puuC+placiD) generated $36.7 \mathrm{~g} / \mathrm{L}$ 3 -HP at 36 h (Fig. 5b), with $41.7 \%$ of glycerol conversion ratio (GCR) and $1.02 \mathrm{~g} / \mathrm{L} / \mathrm{h}$ of productivity (Table 1 ). The GCR is calculated as the each metabolite concentration divided by the total glycerol concentration. We also found that the strain $\mathrm{Kp}(\mathrm{ptac}-$ puuC + placiMALD) harboring CRISPRi vector targeting all four lactate-producing enzyme genes produced $26.9 \mathrm{~g} / \mathrm{L} 3-\mathrm{HP}$ at $48 \mathrm{~h}$ (Fig. 5c), with GCR of $34.6 \%$ and $0.56 \mathrm{~g} / \mathrm{L} / \mathrm{h}$ of productivity (Table 2). Compared with $\mathrm{Kp}($ ptac-puuC + placiD), the strain $\mathrm{Kp}($ ptac-puuC + placiMALD) produced more acetic acid to compensate cofactor for cell growth. For above two strains, the overall conversion ratio from glycerol to major metabolites was around $60-70 \%$, indicating that partial carbon source flowed into other pathways. It should be pointed out that CRISPRi system imposed a burden on cell growth. This finding is consistent with other study [25]. As Fig. 5 shown, the highest $\mathrm{OD}_{600}$ was only $20-30$.

\section{Dissecting interactions of lactate-producing enzyme genes by CRISPRi strains}

A number of enzymes potentially contribute to lactic acid synthesis (Additional file 1: Fig. S1). To decipher their interactions, a total of four CRISPRi strains targeting different lactic acid-producing enzyme genes were subjected to qRT-PCR analysis. Results showed that repressing anyone of lactic acid-producing enzyme genes led to up-regulation or down-regulation of other enzyme genes (Fig. 6). For example, in strain $\mathrm{Kp}($ ptac-puuC + placiL) targeting pmd gene, both $l d h A$ and aldA genes were upregulated, while $m g s A$ gene was less affected (Fig. 6a). By contrast, the $m g s A$ gene was significantly upregulated in both $\mathrm{Kp}(\mathrm{ptac}-\mathrm{puuC}+$ placiD) and $\mathrm{Kp}$ (ptacpuu C+ placiA) (Fig. 6b, c), where ldhA and aldA genes were respectively targeted by CRISPRi. In strain Kp(ptacpuu $C+$ placiM) targeting $m g s A$, both $p m b$ and ald $A$ genes were downregulated (Fig. 6d). 

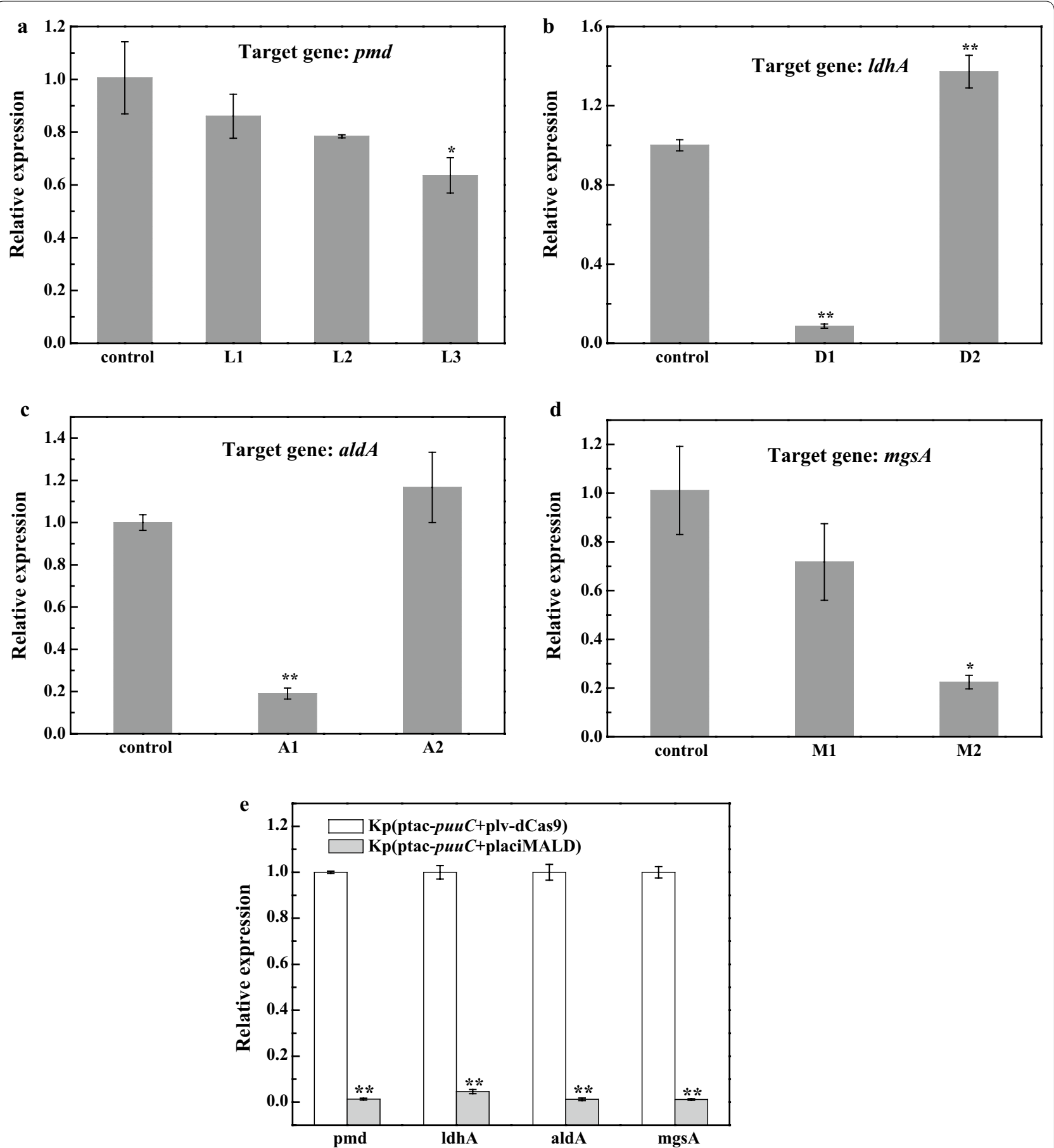

Fig. 3 Relative expression levels of lactate-producing enzyme genes in K. pneumoniae harboring CRISPRi system. a Relative expression level of pmd gene. L1, L2 and L3 indicate three candidate guide RNAs targeting pmd gene. b Relative expression level of IdhA gene. D1 and D2 indicate two candidate guide RNAs targeting IdhA gene. c Relative expression level of aldA gene. A1 and A2 stand for two candidate guide RNAs targeting aldA gene. d Relative expression level of mgsA gene. M1 and M2 refer to two candidate guide RNAs targeting mgsA gene. Control, without guide RNA. e Relative expression levels of pmd, IdhA, aldA and mgsA genes in Kp(ptac-puuC+ placiMALD) at $12 \mathrm{~h}$. Kp(ptac-puuC+plv-dCas9), recombinant $K$. pneumoniae strain harboring vector ptac-puuC and vector plv-dCas9 where Cas 9 was inactive; Kp(ptac-puuC+ placiMALD), recombinant K. pneumoniae strain harboring vector ptac-puUC and CRISPRi vector placiMALD simultaneously targeting four lactate-producing enzyme genes $p m d$, IdhA, ald $A$ and $m g s A$. ${ }^{*} P<0.05 ;{ }^{* *} P<0.01$ 

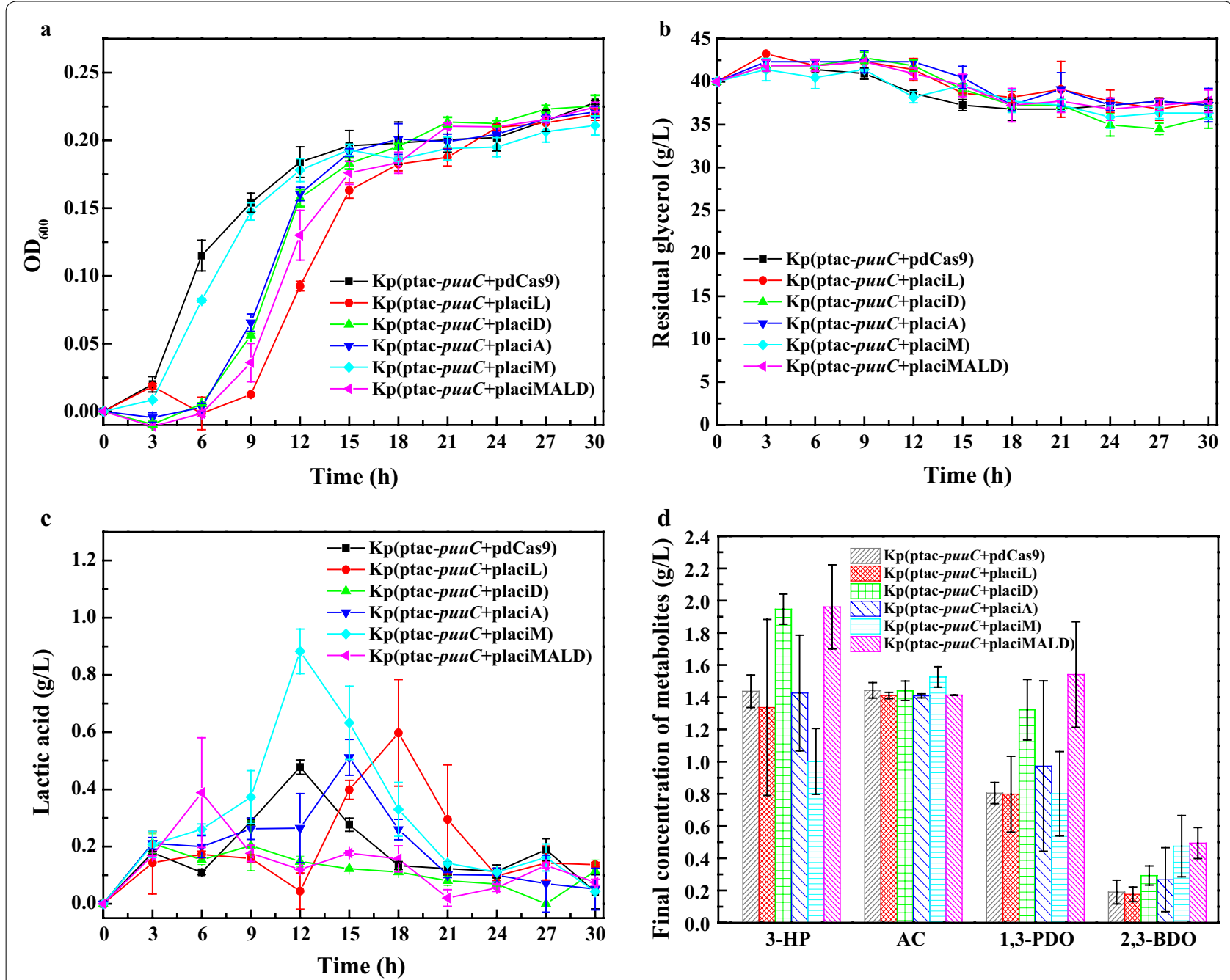

Fig. 4 Shake-flask cultivation of K. pneumoniae strain harboring CRISPRi system. a Growth curve of the recombinant K. pneumoniae strain harboring CRISPRi vector and aldehyde dehydrogenase puuC expression vector. b Residual glycerol concentration. c Lactic acid concentration. $\mathbf{d}$ Metabolites concentration after 30 h cultivation. 3-HP, 3-hydroxypropionic acid; AC, acetic acid; 1,3-PDO, 1,3-propanediol; 2,3-BDO, 2,3-butanediol. placiL, CRISPRi vector targeting L-lactate dehydrogenase gene $\mathrm{pmb}$; placiD, CRISPRi vector targeting $\mathrm{D}$-lactate dehydrogenase gene IdhA; placiA, CRISPRi vector targeting lactaldehyde dehydrogenase gene aldA; placiM, CRISPRi vector targeting methylglyoxal synthase gene mgsA; placiMALD, CRISPRi vector targeting all above four genes. Kp(ptac-puuC + pdCas9), recombinant $K$. pneumoniae strain harboring tac promoter-driven puuC expression vector and dCas9 expression vector; Kp(ptac-puuC + placiL), recombinant K. pneumoniae strain harboring puuC expression vector and CRISPRi vector targeting L-lactate dehydrogenase gene pmb; Kp(ptac-puuC+ placiD), recombinant K. pneumoniae strain harboring puuC expression vector and CRISPRi vector targeting $\mathrm{D}$-lactate dehydrogenase gene IdhA; Kp(ptac-puuC + placiA), recombinant $K$. pneumoniae strain harboring puuC expression vector and CRISPRi vector targeting lactaldehyde dehydrogenase gene alh $A$; Kp (ptac-puuC + placiM), recombinant K. pneumoniae strain carrying puuC expression vector and CRISPRi vector targeting methylglyoxal synthase gene mgsA; Kp(ptac-puuC+ placiMALD), recombinant K. pneumoniae strain harboring puuC expression vector and CRISPRi vector simultaneously targeting four genes mgsA, aldA, pmb and IdhA

\section{Discussion}

In this work, we developed CRISPRi system in K. pneumoniae to attenuate lactic acid formation. The dCas9 was derived from S. pyogenes, and considering the performance of CRISPRi system is largely dependent on guide RNA, we designed several candidate guide RNAs and screened the best. The qRT-PCR results showed that all four lactate-producing enzyme genes were transcriptionally repressed (Figs. 3e, 6). In micro-aerobic conditions, the strain $\mathrm{Kp}($ ptac-puuC+placiD) coexpressing PuuC and ldhA-targeting dCas9-sgRNA complex produced similar level of lactic acid with the strain $\mathrm{Kp}($ ptac-puuC + placiMALD) coexpressing puuC and dCas9-sgRNA targeting four lactate-producing enzyme genes (Figs. 4c, 5b, c), indicating that $l d h A$ is the predominant gene for lactic acid synthesis. Importantly, 

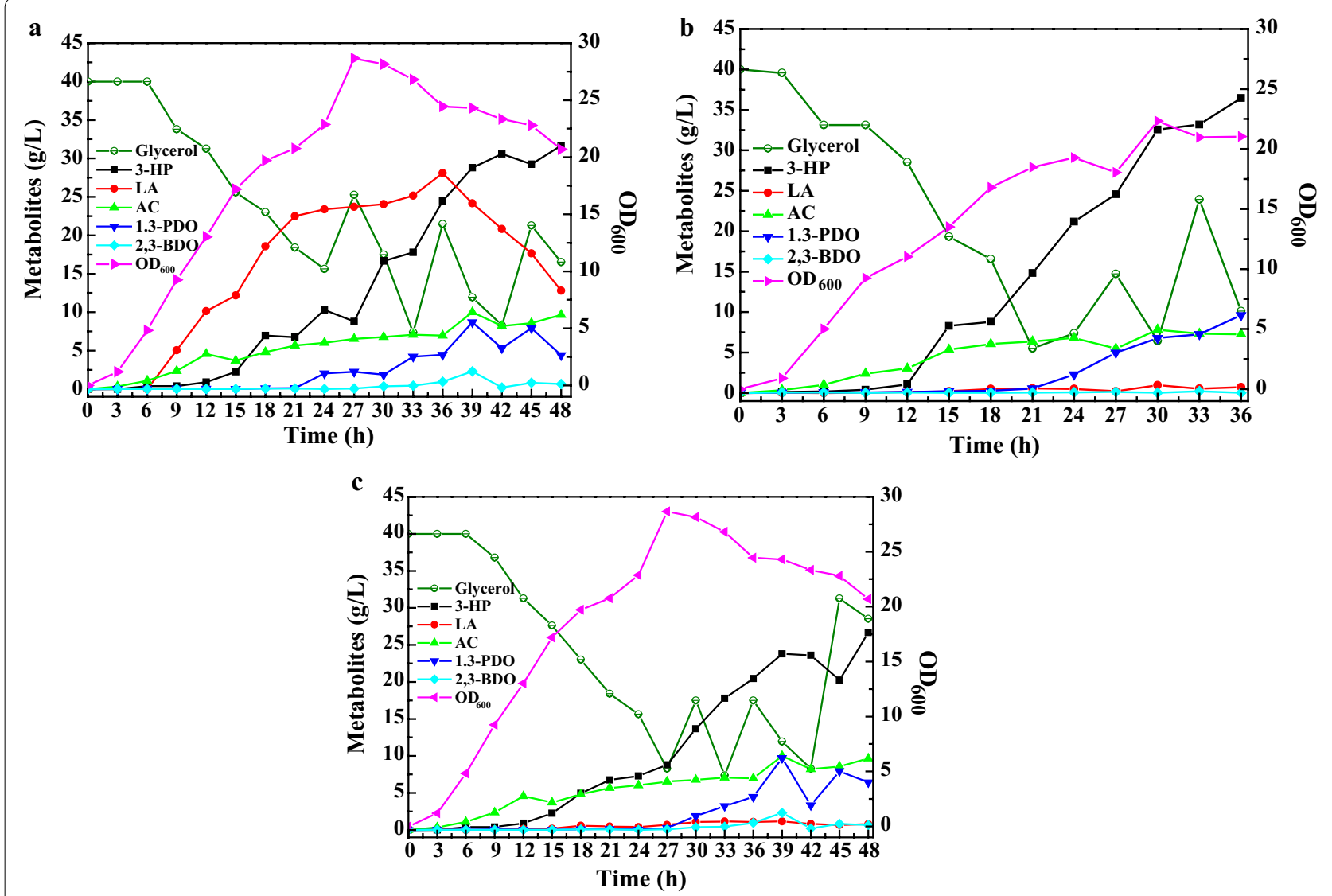

Fig. 5 Bioreactor cultivation of the K. pneumoniae strains harboring CRISPRi system. a Kp(ptac-puuC+pdCas9), recombinant K. pneumoniae strain harboring aldehyde dehydrogenase puuC expression vector and dCas9 expression vector; b Kp(ptac-puuC+ placiD), recombinant K. pneumoniae strain harboring aldehyde dehydrogenase puuC expression vector and CRISPRi vector targeting D-lactate dehydrogenase gene IdhA; c Kp(ptacpuuC + placiMALD), recombinant $K$. pneumoniae strain harboring puuC expression vector and CRISPRi vector targeting four lactate-producing enzyme genes mgsA, aldA, pmb and IdhA. 3-HP, 3-hydroxypropionic acid; LA, lactic acid; AC, acetic acid; 1,3-PDO, 1,3-propanediol; 2, 3-BDO, 2,3-butanediol

Table 1 Carbon distribution of Kp(ptac-puuC + placiD) in a $5 \mathrm{~L}$ bioreactor ( $36 \mathrm{~h}$ )

\begin{tabular}{lllllll}
\hline Metabolites & 3-HP & LA & AA & 1, 3-PDO & 2, 3-BDO & Total \\
\hline Titer (mM) & 407 & 7 & 123 & 126 & 0 & 976 \\
Titer (g/L) & 36.7 & 0.7 & 7.4 & 9.6 & 0 & 54.4 \\
GCR (\%) & 41.7 & 0.7 & 12.6 & 12.9 & 0 & 67.9 \\
\hline
\end{tabular}

3-HP 3-hydroxypropionic acid, LA lactic acid, $A A$ acetic acid, 1,3-PDO 1,3-propanediol, 2,3-BDO 2,3-butanediol, GCR glycerol conversion ratio $\mathrm{Kp}($ ptac-puuC+ placiD), recombinant $K$. pneumoniae strain harboring vector overexpressing puuC and CRISPRi vector targeting IdhA gene

Table 2 Carbon distribution of Kp(ptac-puuC + placiMALD) in a 5 L bioreactor (48 h)

\begin{tabular}{lllllll}
\hline Metabolites & 3-HP & LA & AA & 1,3-PDO & 2,3-BDO & Total \\
\hline Titer (mM) & 298 & 7 & 163 & 84 & 7 & 861 \\
Titer (g/L) & 26.9 & 0.7 & 9.8 & 6.4 & 0.7 & 44.5 \\
GCR (\%) & 34.6 & 0.8 & 18.9 & 9.8 & 0.8 & 64.9 \\
\hline
\end{tabular}

3-HP 3-hydroxypropionic acid, LA lactic acid, $A A$ acetic acid, 1,3-PDO 1,3-propanediol, 2,3-BDO 2,3-butanediol, GCR glycerol conversion ratio

$\mathrm{Kp}($ ptac-puuC + placiD), recombinant $K$. pneumoniae harboring vector overexpressing puuC and CRISPRi vector simultaneously targeting four lactate-producing enzyme genes IdhA, ald $A$, pmd and $m g s A$ 

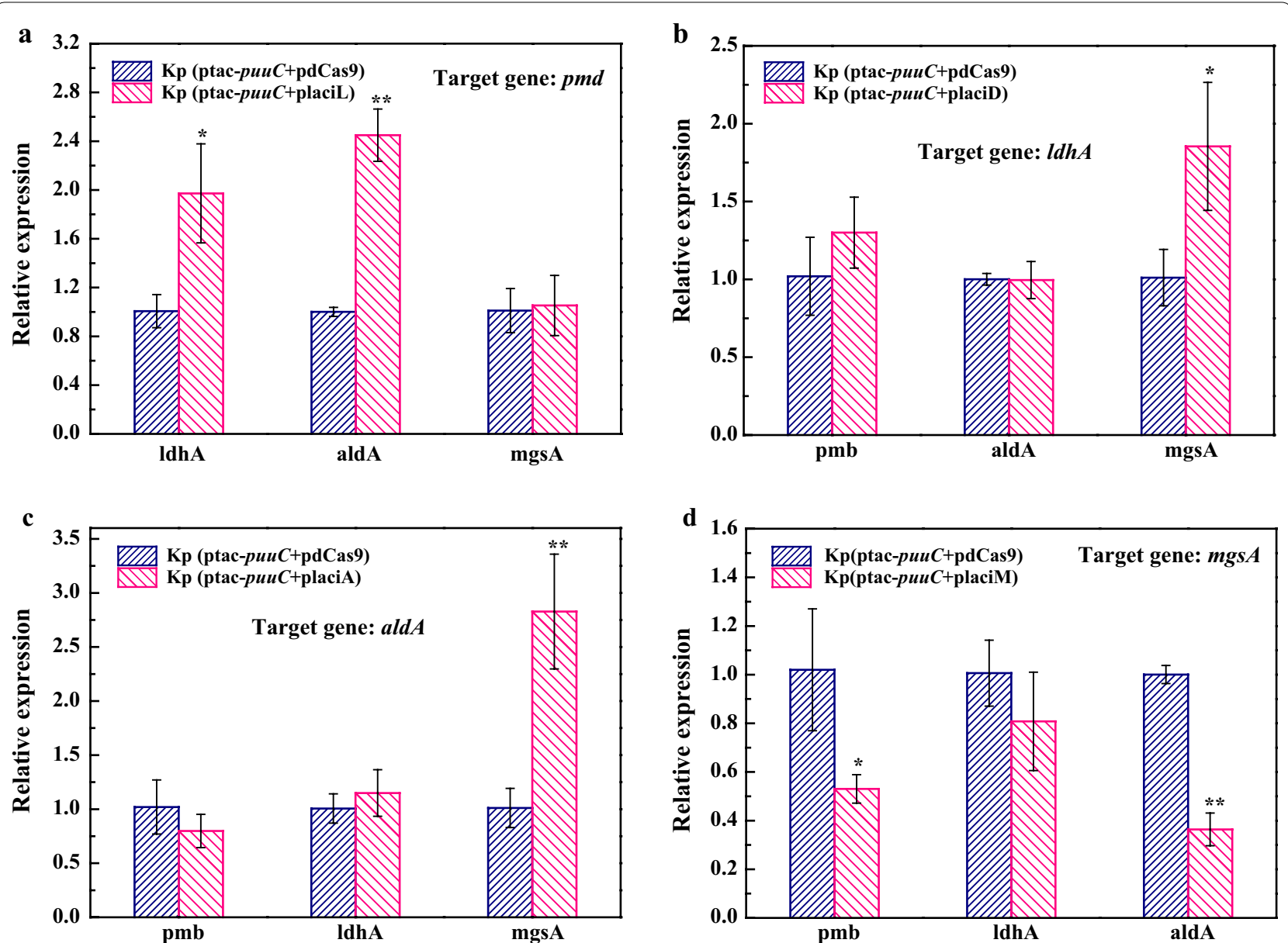

Fig. $\mathbf{6}$ Relative expression levels of lactate-producing enzyme genes in K. pneumoniae strain harboring CRISPRi system. a Relative expression levels of IdhA, aldA and mgsA genes in K. pneumoniae strain harboring CRISPRi system targeting $p m d$ gene; $\mathbf{b}$ Relative expression levels of $p m d$, ald $A$ and mgsA genes in K. pneumoniae strain harboring CRISPRi system targeting IdhA; c Relative expression levels of pmd, IdhA and $m g s A$ genes in K. pneumoniae strain harboring CRISPRi system targeting aldA; d Relative expression levels of $p m d$, IdhA and aldA genes in K. pneumoniae strain harboring CRISPRi system targeting mgsA. Kp(ptac-puuC+ pdCas9), recombinant K. pneumoniae strain harboring aldehyde dehydrogenase puuC expression vector and dCas9 expression vector; $\mathrm{Kp}$ (ptac-puuC+ placiL), recombinant $K$. pneumoniae strain harboring puuC expression vector and CRISPRi vector targeting L-lactate dehydrogenase gene pmb; Kp(ptac-puuC+ placiD), recombinant $K$. pneumoniae strain harboring puuC expression vector and CRISPRi vector targeting D-lactate dehydrogenase gene IdhA; Kp(ptac-puuC+ placiA), recombinant K. pneumoniae strain harboring puuC expression vector and CRISPRi vector targeting lactaldehyde dehydrogenase gene aldA; Kp(ptac-puUC+ placiM), recombinant $K$. pneumoniae strain harboring puuC expression vector and CRISPRi vector targeting methylglyoxal synthase gene $m g s A$. ${ }^{*} P<0.05$; **P $<0.01$

in fed-batch cultivation, although all CRISPRi strains produced similar levels of lactic acid at 24-30 h (Fig. 4c), however the strains $\mathrm{Kp}(\mathrm{ptac}-\mathrm{puuC}+$ placiD $)$ and $\mathrm{Kp}$ (ptac-puuC+placiMALD) generated low levels of lactic acid during entire fermentation process. Clearly, this offers flexibility for fermentation, because lactic acid maintains minimal throughout (Fig. 5b, c). It should be pointed out that leaky expression of dCas9 occurred. This could be partially ascribed to unknown aTc analogs in yeast extract or possible influences of TetR/tetO on aTc. This engineered CRISPRi system needs amelioration prior to real-world application.
Our previous study reported the high titer of 3-HP $(83.8 \mathrm{~g} / \mathrm{L})$ in K. pneumoniae (2), which is higher than CRISPRi strains. The low 3-HP production in CRISPRi strains could be partially attributed to the expression of dCas9 which imposed a heavy burden on cells. Despite low 3-HP production, lactic acid was attenuated by the engineered CRISPRi system, which simplified downstream separation. The remaining lactic acid in bioreactor is limited, and can be converted to other metabolites by expressing an enzyme. Alternatively, lactic acid can be separated using preparative chromatography. Clearly, this is uneconomical for large-scale fermentation. If 3-HP is 
converted to acrylic acid simply by high temperature, the remaining lactic acid may not affect this process. In fact, separation of 3-HP from lactic acid is extremely challenging because they are isomers. So far, nearly no techniques can efficiently separate 3-HP from lactic acid. Lactic acid is mainly generated from pyruvate and its synthesis pathways are conserved in nearly all organisms (Additional file 1: Fig. S1). Conventional approaches to block lactic acid formation rely mainly on the deletion of enzyme genes or optimization of fermentation conditions [2, 7]. However, these approaches neglect the complexity of lactic acid pathways and their contribution to the production of desired metabolites such as 3-HP and 1,3-PDO [9]. From the viewpoint of evolution, the complexity of lactic acid pathways enable bacteria to buffer external stimuli and adapt harsh environment. Deduced from Additional file 1: Fig. S1, deletion of one or two lactic acid pathways might not be lethal to K. pneumoniae owing to tailored compensation mechanism of lactic acid synthesis. That is, repression rather than deletion of lactic acidsynthesizing enzyme genes is appropriate for metabolic engineering purposes. As such, CRISPRi was exploited to decipher and tune lactic acid metabolism.

In this study, the $l d h A$ gene was shown to be pivotal for lactic acid accumulation when glycerol was the sole carbon source and micro-aerobic conditions were maintained. As shown in Figs. 4c, 5b, inhibiting ldhA gene alone almost completely blocked lactic acid synthesis. This may be explained by the following reasons: (i) 3-HP production relies on PuuC overexpression which consumes $\mathrm{NAD}^{+}$and ATP [2]. Since lactic acid biosynthesis is accompanied by the generation of $\mathrm{NAD}^{+}$and ATP, lactic acid was thus synthesized. (ii) Although L-lactate dehydrogenase $(p m b)$ and lactaldehyde dehydrogenase (aldA) catalyze the formation of L-lactic acid (Fig. 1, Additional file 1: Fig. S1), however, the corresponding CRISPRi strains produced more lactic acid compared with the CRISPRi strain targeting D-lactate-producing enzyme gene (Fig. 4c). Presumably, D-lactate dehydrogenase contributed largely to lactic acid level. (iii) It has been reported that lactaldehyde can be synthesized through fucose and rhamnose metabolisms [26] (Additional file 1: Fig. S1) and lactaldehyde may serve as an intermediate to alleviate the cytotoxicity of methylglyoxal. However, CRISPRi-based inactivation of lactaldehyde dehydrogenase (aldA) and methylglyoxal synthase $(m g s A)$ failed to effectively block lactic acid production (Fig. 4c), indicating that lactic acid biosynthesis through methylglyoxal and lactaldehyde pathways may be minimal in current conditions, and methylglyoxal detoxification has little impacts on lactate production and cell growth. Another finding of this study is carbon flux compensation between lactate-producing pathways, as repressing one pathway elicited other pathways (Fig. 6).

CRISPRi can simultaneously repress multiple genes due to recruitment of guide RNAs ([18, 25, 27], Fig. 4c). For enzymes in central pathways, deleting their synthesis genes may impede cell growth or even elicit cell death [5]. CRISPRi is an ideal tool to coordinate cell growth and bioproduction of desired metabolites. Furthermore, unlike RNA interference which represses gene expression at posttranscriptional level, CRISPRi suppresses gene expression at transcriptional level and thus consumes less cellular resources. More importantly, CRISPRi can be easily applied in microbes lacking NHEJ pathway or for which no efficient homologous recombination approach is available. Hence, we believe that the CRISRPi system developed in this study will facilitate functional characterization of key genes and metabolic engineering of $K$. pneumoniae.

\section{Methods}

Strains, medium and chemicals

Strains of K. pneumoniae DSM 2026 and E. coli Top10 were purchased from DSMZ GmbH, Germany. Strain $K$. pneumoniae DSM 2026 was used as the host for development of CRISPRi system. E. coli Top10 was used for gene cloning (Additional file 2: Fig. S2, Additional file 3: Table S1). In vector construction experiments, all strains were grown in LB medium containing the following components per liter: $5 \mathrm{~g}$ yeast extract, $10 \mathrm{~g} \mathrm{NaCl}$, $10 \mathrm{~g}$ peptone, and $50 \mathrm{mg}$ kanamycin or $34 \mathrm{mg}$ chloramphenicol. In CRISPRi experiments, strains were grown in fermentation medium containing the following components per liter: $\mathrm{K}_{2} \mathrm{HPO}_{4} \cdot 3 \mathrm{H}_{2} \mathrm{O}, 3.4 \mathrm{~g} ; \mathrm{KH}_{2} \mathrm{PO}_{4}, 1.3$ g; $\left(\mathrm{NH}_{4}\right)_{2} \mathrm{SO}_{4}, 4 \mathrm{~g} ; \mathrm{MgSO}_{4} \cdot 7 \mathrm{H}_{2} \mathrm{O}, 0.5 \mathrm{~g} ; \mathrm{CaCO}_{3}, 0.1$ g; yeast extract, $3 \mathrm{~g}$; glycerol, $40 \mathrm{~g}$; and $1.25 \mathrm{~mL}$ of trace element solution. The trace element solution contained the following components per liter: $\mathrm{FeSO}_{4}, 32 \mathrm{~g}$; $\mathrm{ZnCl}_{2} \cdot 6 \mathrm{H}_{2} \mathrm{O}$, $2.72 \mathrm{~g} ; \mathrm{MnCl}_{2} \cdot 4 \mathrm{H}_{2} \mathrm{O}, 0.68 \mathrm{~g} ; \mathrm{CoCl}_{2} \cdot 6 \mathrm{H}_{2} \mathrm{O}, 1.88 \mathrm{~g} ; \mathrm{H}_{3} \mathrm{BO}_{3}$, $0.24 \mathrm{~g} ; \mathrm{Na}_{2} \mathrm{MoO}_{4}, 0.02 \mathrm{~g} ; \mathrm{CuCl}_{2} \cdot 2 \mathrm{H}_{2} \mathrm{O}, 1.88 \mathrm{~g}$ and $40 \mathrm{~mL}$ concentrated $\mathrm{HCl}$. Restriction enzymes, taq DNA polymerase and T4 DNA ligase were purchased from TaKaRa (Dalian, China). Primer synthesis and DNA sequencing were performed by Biomed Co., Ltd. Other chemicals for fluorescence assay, gel electrophoresis, and HPLC analysis were products of Sigma.

\section{Construction of recombinants}

To develop CRISPRi system in K. pneunomiae, enhanced green fluorescent protein (EGFP) was used as a reporter. Vector ptac-egfp was constructed by insertion of tac-egfp expression cassette into pET-28a between BglII and XhoI. The CRISPRi vectors targeting egfp gene or lactate-producing enzyme genes were constructed by replacement 
of the sgRNA sequence in vector plv-dCas9-sgRNA [28] (Additional file 2: Fig. S2, Additional file 3: Table S1). Briefly, two complementary single-stranded target sequences were chemically synthesized and annealed to form a $23 \mathrm{bp}$ double-stranded DNA owning cohesive ends matching the BspQI-digested vector. Subsequent ligation resulted in desired CRISPRi vectors. Vector pdCas9 was achieved by deleting the sgRNA sequence in vector plv-dCas9-sgRNA with XmaI/NgoMIV, followed by ligation. To ensure efficient inhibition, two candidate guide RNAs targeting the different regions of EGFP promoter were synthesized and the resultant vectors were designated ptaci $_{1}$ and ptaci $\mathrm{T}_{2}$ (Additional file 3: Table S1). For each lactate-producing enzyme gene, two or three CRISPRi vectors were constructed and named after respective gene (Additional file 4: Table S2). For example, 'placiM' indicates the CRISPRi vector targeting methylglyoxal synthase gene $m g s A$; 'placiD' refers to CRISPRi vector targeting D-lactate dehydrogenase gene ldhA; 'placiL' indicates CRISPR vector targeting L-lactate dehydrogenase (EC:1.1.1.27) gene $p m b$; 'placiA' represents the CRISPR vector targeting lactaldehyde dehydrogenase alhA; while 'placiMALD' indicates CRISPRi vector simultaneously targeting above four genes (Additional file 3 : Table S1).

\section{Electro-transformation and screening}

The Eppendorf tube containing competent $K$. pneunomiae cells was embedded in ice for $30 \mathrm{~min}$ and then centrifuged to harvest cells. The cells were extensively rinsed with cold $\mathrm{ddH}_{2} \mathrm{O}$ to remove ions and subsequently mixed with vectors. The mixture was added into a MicroPulser Cuvette for electroporation $(0.2 \mathrm{~cm}, 2.5 \mathrm{kV}$, time duration $>0.5 \mathrm{~ms}$ ) based on manufacturer's instructions. After cultivation in $\mathrm{LB}$ medium at $37^{\circ} \mathrm{C}$ for $1 \mathrm{~h}$, the cells were plated on LB-agar medium containing chloramphenicol $(34 \mu \mathrm{g} / \mathrm{mL})$, kanamycin $(50 \mu \mathrm{g} / \mathrm{mL})$ or both of them depending on experimental requirements (Additional file 4: Table S2).

\section{Fluorescence assay of CRISPRi strains targeting lactate-producing enzyme genes}

To establish CRISPRi system in K. pneunomiae, a panel of recombinant $K$. pneunomiae strains were constructed with EGFP as a reporter. Briefly, vectors ptac-egfp, placiT $_{1}$, placiT 2 (CRISPRi vectors targeting different region of the promoter sequence of EGFP) (Fig. 2), and the control vector plv-dCas9-sgRNA with non-targeting sgRNA, were individually transformed into component $K$. pneunomiae cells, resulting in recombinant strains $K$. pneunomiae (ptac-egfp), K. pneunomiae (ptac-placi $\left.\mathrm{T}_{1}\right)$, K. pneunomiae(ptac-placi $\mathrm{T}_{2}$ ) and $K$. pneunomiae(plvdCas9-sgRNA), respectively. These recombinant strains were grown in LB medium for $15-20 \mathrm{~h}$, and then transferred to $250 \mathrm{~m}$ : flasks containing $100 \mathrm{~mL}$ fermentation medium, $85 \mu \mathrm{g} / \mathrm{mL}$ chloramphenicol and $50 \mu \mathrm{g} / \mathrm{mL}$ kanamycin. The strains were cultivated in a rotary shaker at $200 \mathrm{rpm}$ and $37^{\circ} \mathrm{C}$. After $3 \mathrm{~h}$ cultivation, IPTG and aTc, at final concentrations of $0.5 \mathrm{mM}$ and $2 \mu \mathrm{M}$, respectively, were added into fermentation broth, and the cultivation conditions were adjusted to $30{ }^{\circ} \mathrm{C}$ and $150 \mathrm{rpm}$ to induce $\mathrm{dCas} 9$ expression. At $18 \mathrm{~h}$, fermentation broth was diluted tenfold to examine $\mathrm{OD}_{600}$ using a visible spectrophotometer (APL instrument, Shanghai). The fermentation broth was directly used for fluorescence assay by a fluorescence spectrophotometer (HITACHI).

\section{Real-time PCR analysis of CRISPRi strains targeting lactate-producing enzyme genes}

Klebsiella pneumoniae cells were harvested by centrifugation at $12,000 \mathrm{rpm}$ and $4{ }^{\circ} \mathrm{C}$ and immediately chilled with liquid nitrogen to avoid RNA degradation. The cells were used for extracting total RNA. For CRISPRi strains $\mathrm{Kp}($ ptac-puuC + placiL), Kp(ptac-puuC+ placiD), $\mathrm{Kp}(\mathrm{ptac}-$ puuC + placiA $), \quad \mathrm{Kp}(\mathrm{ptac}-$ puu $C+$ placiM $)$ and $\mathrm{Kp}($ ptac- $p u u C+$ placiMALD), total RNA was extracted using the RNA prep pure Cell/Bacteria Kit (Tiangen, Beijing, China). The cDNA was synthesized using $\mathrm{HiFi}$ MMLV cDNA Synthesis Kit (CWbio Co. Ltd). The chemically synthesized cDNA was mixed and subjected to gradient dilution and served as template to determine the specificity and efficiency of the primers. qRTPCR was carried out using UltraSYBR mixture (with ROX) (CWbio. Co. Ltd). The cDNA from each sample was diluted to determine the linear range for qRT-PCR (Fig. 3). 16S rRNA was recruited as the internal standard in qRT-PCR analysis. The statistics was analyzed using $2^{-\Delta \Delta C t}$ strategy.

\section{Shake-flask cultivation of CRISPRi strains targeting lactate-producing enzyme genes}

The CRISPRi vectors targeting one or four lactateproducing enzyme genes were transformed into $K$. pneumoniae(ptac-puuC), generating recombinant strains $\mathrm{Kp}(\mathrm{ptac}-p u u C+$ placiM $), \quad \mathrm{Kp}($ ptac- $p u u C+$ placiA), Kp(ptac-puuC+ placiL $), \quad \mathrm{Kp}($ ptac-puuC + placiD $)$ and $\mathrm{Kp}($ ptac-puuC+placiMALD). The strain harboring vector plv-dCas9 was used as a control (Additional file 3: Table S1). All above recombinant $K$. pneumoniae strains were grown in LB medium for $10 \mathrm{~h}$ and subsequently transferred to shake flasks containing fermentation medium, $85 \mu \mathrm{g} / \mathrm{mL}$ chloramphenicol and $50 \mu \mathrm{g} / \mathrm{mL}$ kanamycin. These strains were cultivated at $37^{\circ} \mathrm{C}$ and $150 \mathrm{rpm}$. After $3 \mathrm{~h}$ cultivation, IPTG and aTc at final concentrations of $0.5 \mathrm{mM}$ and $2 \mu \mathrm{M}$, respectively, were added to induce dCas 9 expression. The fermentation broth was 
sampled every $3 \mathrm{~h}$ to examine cell growth, glycerol consumption and metabolites formation.

\section{Bioreactor cultivation of CRISPRi strains targeting lactate-producing enzyme genes}

Strains Kp(ptac-puuC+placiD) and Kp(ptacpuu $C+$ placiMALD) were grown in shake-flasks containing $\mathrm{LB}$ medium at $37{ }^{\circ} \mathrm{C}$ and shaken at $150 \mathrm{rpm}$. After $24 \mathrm{~h}$ cultivation, strains were transferred to $5 \mathrm{~L}$ bioreactor (Baoxing, China) containing antibiotics, IPTG and fermentation medium aforementioned. Fermentation conditions were similar to previously reported [2]. Briefly, agitation speed was $400 \mathrm{rpm}$, air was supplied at $1.5 \mathrm{vvm}, \mathrm{pH}$ was maintained at 7.0 by titration with $5 \mathrm{M}$ $\mathrm{NaOH}$. The initial glycerol concentration was $40 \mathrm{~g} / \mathrm{L}$. Dissolved oxygen was monitored with electrode. Fermentation broth was sampled every $3 \mathrm{~h}$ to examine biomass, residual glycerol and metabolites. Glycerol was supplemented when its concentration dropped below $10 \mathrm{~g} / \mathrm{L}$.

\section{Analytical methods}

Cell concentrations were measured by using microplate reader (Multiskan FC, Thermo) at $600 \mathrm{~nm}$ with $200 \mu \mathrm{L}$ fermentation broth added in a cuvette. To measure metabolites, fermentation broth was centrifuged at $12,000 \mathrm{rpm}$ for $10 \mathrm{~min}$ to remove bacteria. The $3-\mathrm{HP}$, lactic acid and acetic acid in supernatant were analyzed by HPLC (Shimazu, Tokyo, Japan) system equipped with a $\mathrm{C}_{18}$ column and a SPD-20A UV detector at $210 \mathrm{~nm}$. The column was maintained at $25{ }^{\circ} \mathrm{C}$. The mobile phase was $0.05 \%$ phosphoric acid at a flow rate of $0.8 \mathrm{~mL} / \mathrm{min}$. 1,3-PDO and 2,3-BDO were analyzed by GC (Persee). Briefly, the sample were evaporated at $80^{\circ} \mathrm{C}$ for $40 \mathrm{~min}$ to remove water, and then dissolved in ethanol for $\mathrm{GC}$ analysis. Analytical pure of 1,3-PDO and 2,3-BDO were used as standard for quantification.

\section{Additional files}

Additional file 1: Fig. S1. Conserved lactic acid pathways in diverse
species.

Additional file 2: Fig. S2. Schematic diagram of CRISPRi vectors and PuuC expression vector.

Additional file 3: Table S1. Strains and vectors used in this study. Additional file 4: Table S2. Primers for engineering CRISPRi vectors.

\footnotetext{
Abbreviations

EGFP: enhanced green fluorescent protein; 3-HPA: 3-hydroxypropionaldehyde 1,3-PDO: 1,3-propanediol; 2,3-BDO: 2,3-butanediol; 3-HP: 3-hydroxypropionic acid; CRISPRi: CRISPR interference; ALDH: aldehyde dehydrogenase; qRT-PCR: quantitative real-time PCR; GCR: glycerol conversion ratio; GC: gas chromatography; sgRNA: single guide RNA; NHEJ: non-homologous end joining; aTc: anhydrotetracycline; HPLC: high performance liquid chromatography.
}

Authors' contributions

JW and PT conceived and designed the experiments. JW and PZ performed experiments. YL and LX analyzed data. PT wrote the manuscript. All authors read and approved the final manuscript.

\section{Author details}

1 Beijing Key Laboratory of Bioprocess, College of Life Science and Technology, Beijing University of Chemical Technology, Beijing 100029, People's Republic of China. ${ }^{2}$ College of Biochemical Engineering, Beijing Union University, Beijing 100023, People's Republic of China.

\section{Acknowledgements \\ We thank Professor George Guoqiang Chen from Tsinghua University for providing pdCas9 plasmid. We appreciate Geran Tian from Cornell University for polishing of this manuscript.}

\section{Competing interests}

The authors declare that they have no competing interests.

\section{Availability of data and materials}

The datasets supporting the conclusions of this article are included within the article and its additional files.

\section{Consent for publication}

All authors agree to submission and publication.

\section{Ethics approval and consent to participate}

The manuscript does not include any experiments on vertebrates or regulatory invertebrates.

\section{Funding}

This work was supported by grants from National High Technology Research and Development Program (863 Program) (No. 2015AA021003), National Natural Science Foundation of China (Nos. 21276014, 21476011), National Basic Research Program of China (973 Program) (No. 2012CB725200), and Fundamental Research Funds for the Central Universities (YS1407).

\section{Publisher's Note}

Springer Nature remains neutral with regard to jurisdictional claims in published maps and institutional affiliations.

Received: 22 October 2017 Accepted: 31 March 2018

Published online: 05 April 2018

References

1. Zhang Y, LiY, Du C, Liu M, Cao Z. Inactivation of aldehyde dehydrogenase: a key factor for engineering 1,3-propanediol production by Klebsiella pneumoniae. Metab Eng. 2006;8:578-86.

2. Li Y, Wang X, Ge XZ, Tian PF. High production of 3-hydroxypropionic acid in Klebsiella pneumoniae by systematic optimization of glycerol metabolism. Sci Rep. 2016;6:26932.

3. Yang Y, Lin Y, Li L, Linhardt RJ, Yan Y. Regulating malonyl-CoA metabolism via synthetic antisense RNAs for enhanced biosynthesis of natural products. Metab Eng. 2015;29:217-26.

4. Williams TC, Averesch NJH, Winter G, Plan MR, Vickers CE, Nielsen LK, Kromer JO. Quorum-sensing linked RNA interference for dynamic metabolic pathway control in Saccharomyces cerevisiae. Metab Eng. 2015;29:124-34.

5. Wu G, Yan Q, Jones JA, Tang YJ, Fong SS, Koffas MAG. Metabolic burden: cornerstones in synthetic biology and metabolic engineering applications. Trends Biotechnol. 2016;34:652-64.

6. Celinska E. Debottlenecking the 1,3-propanediol pathway by metabolic engineering. Biotechnol Adv. 2010;28:519-30.

7. Ashok S, Raj SM, Ko Y, Sankaranarayanan M, Zhou S, Kumar V, Park S. Effect of puuC overexpression and nitrate addition on glycerol metabolism and anaerobic 3-hydroxypropionic acid production in recombinant Klebsiella pneumoniae $\Delta$ glpK $\triangle$ dhaT. Metab Eng. 2013;15:10-24. 
8. Ashok S, Sankaranarayanan M, Ko Y, Jae KE, Ainala SK, Kumar V, Park S. Production of 3-hydroxypropionic acid from glycerol by recombinant Klebsiella pneumoniae $\Delta$ dhaT $\Delta y q h D$ which can produce vitamin B12 naturally. Biotechnol Bioeng. 2013;110:511-24.

9. Kumar V, Ashok S, Park S. Recent advances in biological production of 3-hydroxypropionic acid. Biotechnol Adv. 2013;31:945-61.

10. Ko Y, Seol E, Sundara Sekar B, Kwon S, Lee J, Park S. Metabolic engineering of Klebsiella pneumoniae J2B for co-production of 3-hydroxypropionic acid and 1,3-propanediol from glycerol: reduction of acetate and other by-products. Bioresour Technol. 2017;244(Pt 1):1096-103.

11. Feng $X$, Jiang L, Han X, Liu X, Zhao Z, Liu H, Xian M, Zhao G. Production of D-lactate from glucose using Klebsiella pneumoniae mutants. Microb Cell Fact. 2017;16(1):209-19.

12. Forage RG, Lin EC. DHA system mediating aerobic and anaerobic dissimilation of glycerol in Klebsiella pneumoniae NCIB 418. J Bacteriol. 1982;151:591.

13. Skraly FA, Lytle BL, Cameron DC. Construction and characterization of a 1,3-propanediol operon. Appl Environ Microbiol. 1998;64:98-105.

14. Xu YZ, Guo NN, Zheng ZM, Ou XJ, Liu HJ, Liu DH. Metabolism in 1,3-propanediol fed-batch fermentation by a D-lactate deficient mutant of Klebsiella pneumoniae. Biotechnol Bioeng. 2009;104(5):965-72.

15. Jiang Y, Chen B, Duan C, Sun B, Yang J, Yang S. Multigene editing in the Escherichia coli genome via the CRISPR-Cas9 system. Appl Environ Microbiol. 2015;81:2506-14.

16. Choi KR, Lee SY. CRISPR technologies for bacterial systems: current achievements and future directions. Biotechnol Adv. 2016;34:1180-209.

17. Mougiakos I, Bosma EF, de Vos WM, van Kranenburg R, van der Oost J. Next generation prokaryotic engineering: the CRISPR-Cas toolkit. Trends Biotechnol. 2016;34:575-87.

18. Kim SK, Seong W, Han GH, Lee DH, Lee SG. CRISPR interference-guided multiplex repression of endogenous competing pathway genes for redirecting metabolic flux in Escherichia coli. Microb Cell Fact. 2017;16:188-202.
19. Zerbini F, Zanella I, Fraccascia D, Konig E, Irene C, Frattini LF, Tomasi M, Fantappie L, Ganfini L, Caproni E, Parri M, Grandi A, Grandi G. Large scale validation of an efficient CRISPR/Cas-based multi gene editing protocol in Escherichia coli. Microb Cell Fact. 2017;16:68-85.

20. Park J, Shin H, Lee SM, Um Y, Woo HM. RNA-guided single/double gene repressions in Corynebacterium glutamicum using an efficient CRISPR interference and its application to industrial strain. Microb Cell Fact. 2018;17:4-13.

21. Bikard D, Jiang W, Samai P, Hochschild A, Zhang F, Marraffini LA. Programmable repression and activation of bacterial gene expression using an engineered CRISPR-Cas system. Nucleic Acids Res. 2013;41:7429-37.

22. Naito Y, Hino K, Bono H, Ui-Tei K. CRISPRdirect: software for designing CRISPR/Cas guide RNA with reduced off-target sites. Bioinformatics. 2015;31:1120-3.

23. Qi LS, Larson MH, Gilbert LA, Doudna JA, Weissman JS, Arkin AP, Lim WA. Repurposing CRISPR as an RNA-guided platform for sequence-specific control of gene expression. Cell. 2013;152:1173-83.

24. Larson MH, Gilbert LA, Wang X, Lim WA, Weissman JS, Qi LS. CRISPR interference (CRISPRi) for sequence-specific control of gene expression. Nat Protoc. 2013:8:2180-96.

25. Westbrook AW, Moo-Young M, Chou CP. Development of a CRISPR-Cas9 tool kit for comprehensive engineering of Bacillus subtilis. Appl Environ Microb. 2016;82(16):4876-95.

26. Baldoma L, Aguilar J. Metabolism of L-fucose and L-rhamnose in Escherichia coli: aerobic-anaerobic regulation of L-lactaldehyde dissimilation. J Bacteriol. 1988;170(1):416-21.

27. Jakociunas T, Bonde I, Herrgard M, Harrison SJ, Kristensen M, Pedersen LE, Jensen MK, Keasling JD. Multiplex metabolic pathway engineering using CRISPR/Cas9 in Saccharomyces cerevisiae. Metab Eng. 2015;28:213-22.

28. LV L, Ren YL, Chen JC, Wu Q, Chen GQ. Application of CRISPRi for prokaryotic metabolic engineering involving multiple genes, a case study: controllable P(3HB-co-4HB) biosynthesis. Metab Eng. 2015;29:160-8.
Ready to submit your research? Choose BMC and benefit from:

- fast, convenient online submission

- thorough peer review by experienced researchers in your field

- rapid publication on acceptance

- support for research data, including large and complex data types

- gold Open Access which fosters wider collaboration and increased citations

- maximum visibility for your research: over 100M website views per year

At $\mathrm{BMC}$, research is always in progress.

Learn more biomedcentral.com/submissions 